\title{
A Summary of the Endocrine Society Clinical Practice Guidelines on Congenital Adrenal Hyperplasia due to Steroid 21-Hydroxylase Deficiency
}

\author{
Phyllis W. Speiser, ${ }^{1}$ Ricardo Azziz, ${ }^{2}$ Laurence S. Baskin, ${ }^{3}$ Lucia Ghizzoni, ${ }^{4}$ Terry W. Hensle, ${ }^{5}$ \\ Deborah P. Merke, ${ }^{6}$ Heino F. L. Meyer-Bahlburg, ${ }^{7}$ Walter L. Miller, ${ }^{3}$ Victor M. Montori, ${ }^{8}$ \\ Sharon E. Oberfield, ${ }^{9}$ Martin Ritzen, ${ }^{10}$ and Perrin C. White ${ }^{11}$ \\ ${ }^{1}$ Division of Pediatric Endocrinology, Cohen Children's Medical Center of New York, Hofstra University School of Medicine, \\ 400 Lakeville Rd., Suite 180, New Hyde Park, NY 11040, USA \\ ${ }^{2}$ Cedars-Sinai Medical Center, Los Angeles, CA, USA \\ ${ }^{3}$ University of California San Francisco, San Francisco, CA, USA \\ ${ }^{4}$ University of Turin, Turin, Italy \\ ${ }^{5}$ Columbia University, New York, NY, USA \\ ${ }^{6}$ National Institutes of Health Clinical Center and The Eunice Kennedy Shriver National Institute of Child Health and \\ Human Development, Bethesda, MD, USA \\ ${ }^{7}$ New York State Psychiatric Institute, Columbia University, New York, NY, USA \\ ${ }^{8}$ Mayo Clinic, Rochester, MN, USA \\ ${ }^{9}$ Children's Hospital of New York-Presbyterian, Columbia University College of Physicians and Surgeons, New York, NY, USA \\ ${ }^{10}$ Karolinska Institute, Stockholm, Sweden \\ ${ }^{11}$ University of Texas Southwestern Medical Center, Dallas, TX, USA
}

Correspondence should be addressed to Phyllis W. Speiser, pspeiser@lij.edu

Received 11 March 2010; Accepted 24 March 2010

Copyright (C) 2010 Phyllis W. Speiser et al. This is an open access article distributed under the Creative Commons Attribution License, which permits unrestricted use, distribution, and reproduction in any medium, provided the original work is properly cited.

Steroid 21-hydroxylase deficiency accounts for about 95\% of cases of congenital adrenal hyperplasia (CAH). Newborns are currently being screened for the classical forms of this disease throughout the United States and in 12 other countries. As such, it seems important to develop the best practice guidelines for treating not only infants and children, but affected adults as well. This report gives a brief overview of the most recent expert opinion and clinical practice guidelines for CAH as formulated by The Endocrine Society Task Force.

\section{Introduction}

The Endocrine Society, along with 6 sister medical societies in North America and Europe, as well as a patient education and support organization, recently sponsored the development of an updated set of guidelines for clinicians treating child and adult patients with congenital adrenal hyperplasia (CAH) due to 21-hydroxylase deficiency [1]. The reader is referred to brief [2] and comprehensive [3] reviews of this disease for background. A "Consensus Statement" had been published jointly by representatives of pediatric endocrine societies in $2002[4,5]$, and the present Task Force made an effort to include more information regarding adult patients. In addition, the 2010 Endocrine Society Guideline had the benefit of panel members who are experts in evidence based medicine, specifically, systematic literature review and metaanalysis. As readers may be aware, there are very few high quality randomized, controlled clinical trials dealing with $\mathrm{CAH}$ treatment. Notwithstanding this obstacle, the Task Force combined the results of meta-analyses in two topical and controversial areas of CAH treatment, with expert's opinions, tempered with valuable judgments to arrive at the recommendations. The guidelines were reviewed and approved sequentially by The Endocrine Society's Clinical 
Guidelines Subcommittee and Clinical Affairs Core Committee, members responding to a web posting, The Endocrine Society Council, and finally by representatives of each of the cosponsoring sister medical societies. At each stage the Task Force incorporated changes in response to written comments. Thus, the document represents the collective wisdom of a large and diverse group of practitioners.

The following is a summary of recommendations presented in each of ten areas of interest. A description of the GRADE system for ranking the quality of medical evidence is reviewed in [6]. In brief, "recommendations" are relatively firmly held and graded " 1 ," whereas less wellsupported "suggestions" are graded " 2 ." The number of $\oplus$ symbols following recommendations and suggestions reflect the strength of the evidence.

\section{Newborn Screening}

The Task Force believes that newborn screening for $\mathrm{CAH}$ due to 21-hydroxylase deficiency should be incorporated into all newborn screening programs $(1 \mid \oplus \oplus \mathrm{OO})$. Ideally, the first-tier screening test, a 17-hydroxyprogesterone (17-OHP) immunoassay, should be standardized to one type of assay. At present most programs use DELFIA. We recommend that norms be stratified by gestational age $(1 \mid \oplus \oplus \mathrm{OO})$, rather than by birthweight [7]. Second-tier confirmatory tests by liquid chromatography/tandem mass spectrometry are recommended to improve $\mathrm{CAH}$ screening's positive predictive value [8], presently hovering around 1\% for most US programs. As of this paper, the throughput of tandem mass spectrometry is not fast enough for this method to be used as a primary screen. Each screening program, region, or country should adopt specific protocols to deal with positive newborn screens $(1 \mid \oplus \oplus \mathrm{OO})$.

\section{Prenatal Treatment of CAH}

Prenatal therapy to minimize virilization of affected female fetuses in empiric protocols with arbitrary fixed doses of dexamethasone administered from the mid-first trimester have been in use for close to three decades $[9,10]$. The lack of adequately detailed and controlled long-term follow-up studies on the offspring of treated pregnancies is disappointing. In the centers with the most experience, fewer than $50 \%$ of mothers and offspring have responded to questionnaires (Meyer-Bahlburg, H, personal communication). The Task Force expressed concern about this, and about the fact that, as presently practiced, 7 unaffected male fetuses must be treated for each affected female. Numerous studies in experimental animal models and retrospective studies of human glucocorticoid (GC)-treated non-CAH pregnancies, have revealed potential adverse effects (summarized in [11]). For these reasons, prenatal therapy should be pursued through protocols approved by Institutional Review Boards at centers capable of collecting outcomes data on a sufficiently large number of patients so that risks and benefits of this treatment can be defined more precisely $(2 \mid \oplus \oplus \mathrm{OO})$. To this end, data from multicenter studies with similar protocols should be pooled. Noninvasive determination of fetal sex from fetal cell-free DNA in the mother's blood could help avoid treatment of male fetuses. The latter technique is widespread in Europe, but has not yet been approved for commercial use in the United States. Additionally, it would be of interest to determine whether the dexamethasone dose could be reduced later in gestation to improve safety without sacrificing efficacy.

\section{Diagnosis of NCCAH/CAH after Infancy}

An early morning (before 0800) baseline serum 17-OHP by liquid chromatography/tandem mass spectrometry appears to be the screening test of choice in symptomatic individuals after infancy $(1 \mid \oplus \oplus \mathrm{OO})$. A screening serum 17-OHP greater than $6 \mathrm{nmol} / \mathrm{l}$ or $200 \mathrm{ng} / \mathrm{dl}$ [12] warrants obtaining a complete adrenocortical profile following cosyntropin stimulation to differentiate 21-hydroxylase deficiency from other enzyme defects and to make the diagnosis in borderline cases $(1 \mid \oplus \oplus \mathrm{OO})$. Genotyping is now widely available and is helpful when results of the adrenocortical profile following cosyntropin stimulation test are equivocal, or for purposes of genetic counseling $(2 \mid \oplus \mathrm{OOO})$.

\section{Medical Treatment of CAH in Growing Patients}

The GC of first choice for maintenance therapy of children with $\mathrm{CAH}$ is hydrocortisone [13] in tablet form. For infants, tablets may be crushed, weighed, and mixed with a small amount of liquid and delivered immediately by medication syringe, rather than from bulk suspension preparations that deliver uneven doses $(1 \mid \oplus \oplus \oplus \mathrm{O})$. It is recognized that prednisolone and dexamethasone are sometimes useful in treating patients refractory to hydrocortisone [14], however, the Task Force recommends against the routine chronic use of long-acting potent GCs in growing patients $(1 \mid \oplus \oplus \mathrm{OO})$. All patients with classic $\mathrm{CAH}$ should receive mineralocorticoid (MC) supplementation in the form of oral fludrocortisone and sodium chloride supplements in the newborn period and early infancy, and as needed throughout life $(1 \mid \oplus \oplus \mathrm{OO})$.

GC dosage should be increased in stressful situations such as febrile illness $\left(>38.5^{\circ} \mathrm{C}\right)$, gastroenteritis with dehydration, surgery accompanied by general anesthesia, and major trauma $(1 \mid \oplus \oplus \mathrm{OO})$. We recommend against the use of increased GC doses in mental and emotional stress, minor illness, and before physical exercise, as this would greatly increase the frequency of supraphysiologic dosing $(1 \mid \oplus \mathrm{OOO})$. We also recommend against the use of stress doses of GC in patients with nonclassic CAH unless their adrenal function is suboptimal or iatrogenically suppressed $(1 \mid \oplus \mathrm{OOO})$. We suggest that patients who require treatment always wear or carry medical identification indicating that they have adrenal insufficiency $(2 \mid \oplus$ OOO $)$.

Patients should be regularly monitored for signs of GC excess, inadequate androgen suppression in cases of inadequate GC treatment, or hypertension with excess MC and/or sodium $(1 \mid \oplus \oplus \mathrm{OO})$. We recommend monitoring 
treatment by consistently timed hormone measurements $(1 \mid \oplus \mathrm{OOO})$. Endogenous adrenal steroid secretion should not be completely suppressed in order to avoid adverse effects of overtreatment $(1 \mid \oplus \oplus \mathrm{OO})$. In addition to hormone measurements, we suggest regular monitoring of height, weight, and physical examination; annual bone age X-ray assessment is also suggested after 2 years of age $(2 \mid \oplus \mathrm{OOO})$.

\section{Treatment of NCCAH in Childhood}

Nonclassic CAH (NCCAH) is not generally considered an absolute indication for glucocorticoid or mineralocorticoid replacement therapy. Children diagnosed with NCCAH should be offered treatment when they have inappropriately early onset and rapid progression of pubarche or bone age. Adolescent females with overt virilization or erratic menses may also benefit from treatment $(2 \mid \oplus \oplus \mathrm{OO})$. Asymptomatic individuals with NCCAH need not be treated $(1 \mid \oplus \oplus \mathrm{OO})$. Moreover, previously treated NCCAH patients should be given the option of discontinuing therapy when their symptoms resolve $(2 \mid \oplus \oplus \mathrm{OO})$.

\section{Complications of CAH}

All GC-treated patients should be monitored for iatrogenic Cushing syndrome $(1 \mid \oplus \oplus \mathrm{OO})$. Elements of the visit helpful in this regard are the growth chart for height and weight in children, distribution of body fat, presence of pigmented striae, blood pressure measurements, and blood glucose determinations. Since osteopenia and osteoporosis are rare in pediatric CAH patients, routine evaluation of bone mineral density is discouraged in children, but should be considered in any patient who has been subjected to chronic high doses of glucocorticoids or who has sustained fractures $(2 \mid \oplus \mathrm{OOO})$. Adrenal nodules have been identified more frequently in $\mathrm{CAH}$ patients and carriers than in the general population [15], however, adrenal imaging (generally CT scans) ought to be reserved for those patients who have an atypical clinical or biochemical course due to the high radiation burden of frequent screening $(2 \mid \oplus \mathrm{OOO})$. Males with classic $\mathrm{CAH}$ should be periodically screened with ultrasound for testicular adrenal rests [16]. The latter can often be managed medically by more effectively suppressing the pituitary-adrenal axis.

\section{Feminizing Surgery}

The vast majority of genetic females affected with classic $\mathrm{CAH}$ have female gender identity and behavior, tomboyish play and male occupational preferences notwithstanding $[17,18]$. In addition, most affected women can bear healthy offspring, if desired [19]. This rationale is the basis for the suggestion that severely virilized (Prader stage $\geq 3$ ) females be considered for clitoral and perineal reconstruction in infancy. This type of surgery should only be performed by experienced surgeons in centers with similarly experienced pediatric endocrinologists, mental health professionals, and social work services $(2 \mid \oplus \oplus \mathrm{OO})$. At present, the procedures favored by pediatric urologists are neurovascular-sparing clitoroplasty and vaginoplasty using total or partial urogenital mobilization [20] $(2 \mid \oplus \mathrm{OOO})$. Continued long-term outcome studies of early surgery are to be encouraged.

\section{Alternative Therapies}

Preservation of statural growth potential is an important goal for clinicians caring for CAH children. Suboptimal height outcomes are potentially related to late diagnosis and treatment, overtreatment with glucocorticoids, or nonadherence to the medical regimen. It is eminently possible to achieve adult height within the normal range with standard steroid therapy alone (mean height -1.0 SD corrected for parental height among $>1000$ published cases [Muthusamy K et al. JCEM, in press, 2010]). Thus, the Task Force recommends against the use of alternative treatment approaches, for example, growth hormone and/or treatment to delay puberty or epiphyseal fusion for most children with $\mathrm{CAH}(1 \mid \oplus \oplus \mathrm{OO})$. Children with predicted height $\mathrm{SD} \leq-2.25$ may be considered for such growth-promoting treatments in appropriately controlled trials $(2 \mid \oplus \mathrm{OOO})$. Further prospective, randomized, and carefully controlled studies would be helpful in determining whether the use of growth-promoting drugs increases adult height in patients with $\mathrm{CAH}(2 \mid \oplus \mathrm{OOO})$.

Unlike endogenous continuously variable cortisol secretion, current glucocorticoid replacement therapy is given between once and three times daily. It would therefore be desirable to develop new treatment approaches that could both minimize daily GC exposure and more closely mimic the physiologic state. Recent research efforts along these lines have included a long-acting oral form of hydrocortisone [21] and use of continuous subcutaneous hydrocortisone infusion by insulin pump [22].

Bilateral adrenalectomy has been considered in selected individuals who have failed medical therapy, especially in adult females with SW CAH and infertility [23]. In all such cases, the risk for noncompliance with postoperative replacement therapy must be evaluated prior to surgery. The Task Force advises against routine adrenalectomy to treat $\mathrm{CAH}(2 \mid \oplus \mathrm{OOO})$.

\section{CAH in Adulthood}

As in younger subjects, when the diagnosis of NCCAH is suspected, screening may be done with an early morning serum measurement of 17OHP by liquid chromatography and tandem mass spectrometry. Menstruating women should be screened in the early follicular phase of the menstrual cycle [24]. If equivocal, the result should be confirmed by performing a cosyntropin stimulation test before ordering genotyping $(1 \mid \oplus \oplus \mathrm{OO})$. As discussed above, only individuals with a significant degree of hyperandrogenism or those who desire fertility need to be treated $(2 \mid \oplus \mathrm{OOO})$. CAH women should be screened for their gynecological history, and pelvic examination should be performed beginning in adolescence. Pelvic ultrasound should be reserved for CAH patients with irregular menstrual cycles $(2 \mid \oplus \mathrm{OOO})$. 
Whereas adult males with classic $\mathrm{CAH}$ should continue their glucocorticoids indefinitely, men with NCCAH seldom require treatment. Any $\mathrm{CAH}$ patient with impaired fertility should consult a reproductive endocrinologist and/or fertility specialist $(2 \mid \oplus \oplus \mathrm{OO})$.

In the adult population, long-acting glucocorticoids such as prednisolone and dexamethasone may be used judiciously without concern about growth inhibition, but patients should be monitored at least yearly for iatrogenic Cushing syndrome. Clues to overtreatment include centripetal obesity, striae, hypertension, glucose intolerance, and low bone density.

Genetic counseling should be given to parents of known $\mathrm{CAH}$ patients preconceptually, or at birth of a first $\mathrm{CAH}$ child, and to affected adolescents at the transition to adult care $(1 \mid \oplus \mathrm{OOO})$. A multidisciplinary team consisting of pediatric and adult endocrinologists, reproductive endocrinologists, gynecologists, and urologists is the ideal setting for transitioning $\mathrm{CAH}$ patients to adult care $(2 \mid \oplus \mathrm{OOO})$.

\section{Pregnancy}

Pregnant women with $\mathrm{CAH}$ are best followed jointly by endocrinologists and obstetricians, while continuing their prepregnancy doses of hydrocortisone/prednisolone and fludrocortisone therapy. GC doses often need to be increased during pregnancy, and should be adjusted before symptoms and signs of GC insufficiency occur. The usual hormone measurements to assess control are not informative during pregnancy. Stress doses of hydrocortisone should be used during labor and delivery. Dexamethasone should not be given to pregnant women with $\mathrm{CAH}$, if the mother is the treatment target. This drug is not inactivated by placental 11hydroxysteroid dehydrogenase type 2 , and as such may cause fetal adrenal suppression $(1 \mid \oplus \oplus \mathrm{OO})$.

\section{Mental Health}

It is suggested that patients with $\mathrm{CAH}$ and psychosocial problems associated with disorders of sexual development be referred to mental health staff with specialized expertise in managing such problems. Toward this goal, the Task Force suggests the development, evaluation, and implementation of valid $\mathrm{CAH}$-specific quality of life assessment tools $(2 \mid \oplus \mathrm{OOO})$.

\section{References}

[1] P. W. Speiser, R. Azziz, L. S. Baskin et al., "Congenital adrenal hyperplasia due to steroid 21-hydroxylase deficiency: An Endocrine Society Clinical Practice Guideline," Journal of Clinical Endocrinology \& Metabolism, vol. 95, no. 9, pp. 41334160, 2010.

[2] P. W. Speiser and P. C. White, "Congenital adrenal hyperplasia," The New England Journal of Medicine, vol. 349, no. 8, pp. 776-788, 2003.

[3] P. C. White and P. W. Speiser, "Congenital adrenal hyperplasia due to 21-hydroxylase deficiency," Endocrine Reviews, vol. 21, no. 3, pp. 245-291,2000.
[4] P. E. Clayton, W. L. Miller, S. E. Oberfield, et al., "Consensus statement on 21-hydroxylase deficiency from the European Society for Paediatric Endocrinology and the Lawson Wilkins Pediatric Endocrine Society," Hormone Research, vol. 58, no. 4, pp. 188-195, 2002.

[5] P. E. Clayton, S. E. Oberfield, E. Martin Ritzen, et al., "Consensus: consensus statement on 21-hydroxylase deficiency from the Lawson Wilkins Pediatric Endocrine Society and the European Society for Pediatric Endocrinology," Journal of Clinical Endocrinology and Metabolism, vol. 87, no. 9, pp. 4048-4053, 2002.

[6] B. A. Swiglo, M. H. Murad, H. J. Schünemann, et al., "A case for clarity, consistency, and helpfulness: state-of-theart clinical practice guidelines in endocrinology using the grading of recommendations, assessment, development, and evaluation system," Journal of Clinical Endocrinology and Metabolism, vol. 93, no. 3, pp. 666-673, 2008.

[7] H. J. van der Kamp, C. G. M. Oudshoorn, B. H. Elvers, et al., "Cutoff levels of 17- $\alpha$-hydroxyprogesterone in neonatal screening for congenital adrenal hyperplasia should be based on gestational age rather than on birth weight," Journal of Clinical Endocrinology and Metabolism, vol. 90, no. 7, pp. 3904-3907, 2005.

[8] D. Matern, S. Tortorelli, D. Oglesbee, D. Gavrilov, and P. Rinaldo, "Reduction of the false-positive rate in newborn screening by implementation of MS/MS-based second-tier tests: the Mayo Clinic experience (2004-2007)," Journal of Inherited Metabolic Disease, vol. 30, no. 4, pp. 585-592, 2007.

[9] M. G. Forest and H. G. Dörr, "Prenatal therapy in congenital adrenal hyperplasia due to 21-hydroxylase deficiency: retrospective follow-up study of 253 treated pregnancies in 215 families," Endocrinologist, vol. 13, no. 3, pp. 252-259, 2003.

[10] M. I. New, A. Carlson, J. Obeid, et al., "Extensive personal experience: prenatal diagnosis for congenital adrenal hyperplasia in 532 pregnancies," Journal of Clinical Endocrinology and Metabolism, vol. 86, no. 12, pp. 5651-5657, 2001.

[11] P. W. Speiser and W. L. Miller, "Prenatal treatment of classic CAH with dexamethasone: pro vs. con," Endocrine News, pp. 14-18, April 2008.

[12] M. Bidet, C. Bellanne-Chantelot, M.-B. Galand-Portier, et al., "Clinical and molecular characterization of a cohort of 161 unrelated women with nonclassical congenital adrenal hyperplasia due to 21-hydroxylase deficiency and 330 family members," Journal of Clinical Endocrinology and Metabolism, vol. 94, no. 5, pp. 1570-1578, 2009.

[13] W. Bonfig, S. Bechtold, H. Schmidt, D. Knorr, and H. P. Schwarz, "Reduced final height outcome in congenital adrenal hyperplasia under prednisone treatment: deceleration of growth velocity during puberty," Journal of Clinical Endocrinology and Metabolism, vol. 92, no. 5, pp. 1635-1639, 2007.

[14] S. A. Rivkees and J. D. Crawford, "Dexamethasone treatment of virilizing congenital adrenal hyperplasia: the ability to achieve normal growth," Pediatrics, vol. 106, no. 4, pp. 767$773,2000$.

[15] S. Jaresch, E. Kornely, H.-K. Kley, and R. Schlaghecke, "Adrenal incidentaloma and patients with homozygous or heterozygous congenital adrenal hyperplasia," Journal of Clinical Endocrinology and Metabolism, vol. 74, no. 3, pp. 685-689, 1992.

[16] H. L. Claahsen-van der Grinten, F. C. G. J. Sweep, J. G. Blickman, A. R. M. M. Hermus, and B. J. Otten, "Prevalence 
of testicular adrenal rest tumors in male children with congenital adrenal hyperplasia due to 21-hydroxylase deficiency," European Journal of Endocrinology, vol. 157, no. 3, pp. 339344, 2007.

[17] S. A. Berenbaum and J. M. Bailey, "Effects on gender identity of prenatal androgens and genital appearance: evidence from girls with congenital adrenal hyperplasia," Journal of Clinical Endocrinology and Metabolism, vol. 88, no. 3, pp. 1102-1106, 2003.

[18] H. F. L. Meyer-Bahlburg, C. Dolezal, S. W. Baker, A. A. Ehrhardt, and M. I. New, "Gender development in women with congenital adrenal hyperplasia as a function of disorder severity," Archives of Sexual Behavior, vol. 35, no. 6, pp. 667684, 2006.

[19] K. Hagenfeldt, P. O. Janson, G. Holmdahl, et al., "Fertility and pregnancy outcome in women with congenital adrenal hyperplasia due to 21-hydroxylase deficiency," Human Reproduction, vol. 23, no. 7, pp. 1607-1613, 2008.

[20] R. C. Rink, P. D. Metcalfe, M. P. Cain, K. K. Meldrum, M. A. Kaefer, and A. J. Casale, "Use of the mobilized sinus with total urogenital mobilization," Journal of Urology, vol. 176, no. 5, pp. 2205-2211, 2006.

[21] S. Verma, C. Vanryzin, N. Sinaii, et al., "A pharmacokinetic and pharmacodynamic study of delayed- and extended-release hydrocortisone (Chronocort ${ }^{\mathrm{TM}}$ ) vs. conventional hydrocortisone $\left(\right.$ Cortef $^{\mathrm{TM}}$ ) in the treatment of congenital adrenal hyperplasia," Clinical Endocrinology, vol. 72, no. 4, pp. 441447, 2010.

[22] S. M. Bryan, J. W. Honour, and P. C. Hindmarsh, "Management of altered hydrocortisone pharmacokinetics in a boy with congenital adrenal hyperplasia using a continuous subcutaneous hydrocortisone infusion," Journal of Clinical Endocrinology and Metabolism, vol. 94, no. 9, pp. 3477-3480, 2009.

[23] J. J. Van Wyk and E. M. Ritzen, "The role of bilateral adrenalectomy in the treatment of congenital adrenal hyperplasia," Journal of Clinical Endocrinology and Metabolism, vol. 88, no. 7, pp. 2993-2998, 2003.

[24] R. Azziz, L. A. Hincapie, E. S. Knochenhauer, D. Dewailly, L. Fox, and L. R. Boots, "Screening for 21-hydroxylasedeficient nonclassic adrenal hyperplasia among hyperandrogenic women: a prospective study," Fertility and Sterility, vol. 72, no. 5, pp. 915-925, 1999. 